\title{
Plasma phospholipid fatty acids, dietary fatty acids and prostate cancer risk
}

Julie K. Bassett ${ }^{1}$, Gianluca Severi ${ }^{1,2}$, Allison M. Hodge ${ }^{1}$, Robert J MacInnis ${ }^{1,2}$, Robert A. Gibson ${ }^{3}$, John L. Hopper ${ }^{2}$,

Dallas R. English ${ }^{1,2}$, Graham G. Giles ${ }^{1,2,4}$

${ }^{1}$ Cancer Epidemiology Centre, Cancer Council Victoria, 1 Rathdowne Street, Carlton, Victoria 3053, Australia.

${ }^{2}$ Centre for Molecular, Environmental, Genetic and Analytic Epidemiology, Melbourne School of Population and Global Health, University of Melbourne, 207 Bouverie Street, Carlton, Victoria 3053, Australia.

${ }^{3}$ FOODplus Research Centre, Waite Campus, The University of Adelaide, PMB 1, Glen Osmond, South Australia 5064, Australia.

${ }^{4}$ Department of Epidemiology and Preventive Medicine, Monash University, Melbourne, Australia.

Running title: Plasma phospholipid fatty acids, dietary fatty acids and prostate cancer risk

Corresponding author:

Dr Julie Basset

Phone: +61 39635 5372, Fax: +61 396355330

e-mail: julie.bassett@cancervic.org.au

Keywords: fatty acids, prostate cancer, cohort study

Abbreviations used: ALA: $\alpha$-linolenic acid; CI: confidence interval; CVD: cardiovascular disease; DGLA: dihomo- $\gamma$ linolenic acid; DHA: docosahexaenoic acid; DPA: docosapentaenoic; EPA: eicosapentaenoic acid; FFQ: food frequency questionnaire; HR: hazard ratio; MCCS: Melbourne Collaborative Cohort Study; MUFA: monounsaturated fatty acid; PPL: plasma phospholipid; PUFA: polyunsaturated fatty acid; Q: quintile; SFA: saturated fatty acid; Trans: trans fatty acid

Article category: Epidemiology

Novelty and impact: Animal and experimental studies have demonstrated that long chain $n-3$ fatty acids inhibit the development of prostate cancer, whereas n-6 fatty acids might promote it, but the epidemiological literature has generally reported null associations. Our findings suggest that a lower percentage of saturated fatty acids and higher percentage of oleic acid in plasma phospholipids might reduce prostate cancer risk; an increased risk was observed for dietary linoleic acid.

Conflict of interest: The authors declare that they have no conflict of interest.

Word count:

Abstract:

230

Total word count (introduction-discussion): 3757

Tables:

4

References: 50

This article has been accepted for publication and undergone full peer review but has not been through the copyediting, typesetting, pagination and proofreading process which may lead to differences between this version and the Version of Record. Please cite this article as an 'Accepted Article', doi: 10.1002/ijc.28203 


\section{Abstract}

Animal and experimental studies have demonstrated that long chain n-3 fatty acids inhibit the development of prostate cancer, whereas n-6 fatty acids might promote it. We performed a case-cohort analysis within the Melbourne Collaborative Cohort Study using a random sample of 1,717 men and 464 prostate cancer cases to investigate associations between fatty acids assessed in plasma phospholipids (PPL) or diet (estimated using a 121-item food frequency questionnaire) and prostate cancer risk. Hazard ratios (HRs) and 95\% confidence intervals (CI) were estimated using Cox regression. Prostate cancer risk was positively associated with \%PPL saturated fatty acids (SFA); HR $[95 \% \mathrm{CI}]=1.51[1.06,2.16]$, (Q5 vs. Q1, fifth vs. first quintile); P-trend=0.003. HRs (Q5-Q2 vs. Q1) were significantly elevated for \%PPL palmitic acid. \%PPL oleic acid was inversely associated with risk, HR=0.62 [0.43, 0.91] (Q5 vs. Q1); P-trend=0.04. No statistically significant linear trends were observed for dietary intakes. The HRs were elevated for moderate intakes of linoleic acid (Q2 and Q3 vs. Q1, 1.58 [1.10, 2.28] and 1.70 [1.18, 2.46], respectively), but the increase was not significant for higher intakes (Q4 and Q5). No association varied significantly by tumour aggressiveness (all P-homogeneity $>0.1$ ). Prostate cancer risk was positively associated with \%PPL SFA, largely attributable to palmitic acid and inversely associated with \%PPL monounsaturated fatty acids, largely attributable to oleic acid. Higher risks were also observed for dietary n-6 polyunsaturated fats, primarily linoleic acid.

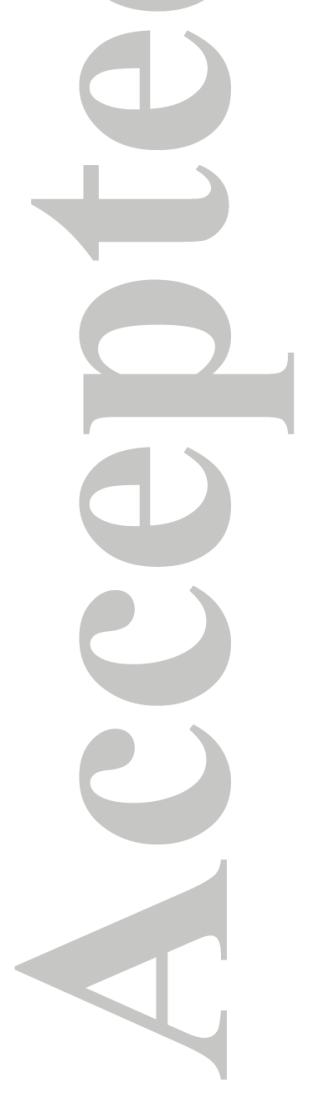




\section{Introduction}

Dietary fat has been widely studied in relation to prostate cancer, but there is only weak evidence for an increased risk associated with total fat or saturated fatty acids (SFA) ${ }^{1,2}$. Reviews have not identified any clear associations with monounsaturated (MUFA) ${ }^{1,2}$, long chain n-3 polyunsaturated (PUFA) or marine fatty acids ${ }^{1,3-8}$ or linoleic acid ${ }^{1-3,9}$ but have reported some evidence of positive associations with intakes or biomarkers of $\alpha$-linolenic acid ${ }^{1,3,4,10}$. Metaanalyses also report wide heterogeneity across studies so pooled estimates must be interpreted cautiously.

Despite generally null epidemiological findings, animal and in vitro experiments have demonstrated that long chain n-3 marine fatty acids, particularly eicosapentaenoic (EPA) and docosahexaenoic acid (DHA) suppress the development of prostate cancer, while n-6 PUFAs possibly promote carcinogenesis ${ }^{11}$. Biological mechanisms have been postulated for dietary long chain n-3 fatty acids in cancer prevention and include suppression of eicosanoid production from arachidonic acid (an n-6 fatty acid metabolised in the body from linoleic acid); influences on transcription factor activity, gene expression, and signal transduction pathways; modulation of estrogen metabolism; increased or decreased production of free radicals and reactive oxygen species; and influences on insulin sensitivity and membrane fluidity ${ }^{8,12}$. Diet, in particular the amount of individual fatty acids, is difficult to measure in epidemiological studies, thus the use of biomarkers of intake is an important adjunct to self-reported intake data. We used a prospective cohort study to investigate associations between both plasma phospholipid (PPL) profiles and dietary intakes of fatty acids and prostate cancer risk.

\section{Materials and methods}

The Melbourne Collaborative Cohort Study (MCCS) is a prospective cohort study of 41,514 people (17,045 men, 24,469 women) recruited between 1990 and 1994 aged 27 to 80 years (99.3\% aged 40 to 69 years). The MCCS study protocol and participants have been described in detail elsewhere ${ }^{13}$. The study protocol was approved by The Cancer Council Victoria’s Human Research Ethics Committee.

\section{The case-cohort study}

We excluded men with a pre-baseline diagnosis of any cancer, a history of angina, myocardial infarction, diabetes or stroke as this may have resulted in dietary change. We also excluded men who reported extreme values of total energy intake $\left(<1^{\text {st }}\right.$ percentile or $>99^{\text {th }}$ percentile), were missing data for any potential confounder, or had insufficient 
information to determine tumour aggressiveness. Of the total 17,045 male participants, this left 14,054 eligible. The case-cohort study included all men diagnosed with prostate cancer between baseline and 30 June 2002 and a random sample (subcohort) of all eligible men. Cases were identified from notifications of first diagnoses of prostate cancer to the Victorian Cancer Registry or the Australian Cancer Database. Aggressive tumours were defined as having a Gleason score $>7$, poor differentiation, stage 4 or fatal disease.

( )

In an average of 8.9 person-years of follow-up to 30 June 2002, 487 eligible men were diagnosed with prostate cancer, 37 left Australia and 752 died from any cause. The subcohort included a random sample of 1784 men, 59 of whom were also cases. \%PPL fatty acid measurements were available for $96 \%$ of members of the subcohort and $95 \%$ of cases. There were no appreciable differences in demographics, lifestyle factors or family history of cancer between men who had their fatty acids measured and those who did not (all $\mathrm{P}>0.06$ ). Thus, the analyses included 1717 men in the subcohort and 464 men with prostate cancer (56 of whom were in the subcohort), including 127 with aggressive tumours.

Samples for participants in the sub-cohort were selected in random order and aliquots of $200 \mu \mathrm{L}$ in batches of 80 samples each (72 study samples and 8 control samples) were prepared. Fatty acid levels were measured in the laboratory of one of the authors (RAG), blind to case status. The \%PPL fatty acid assay has been described in detail elsewhere ${ }^{14}$. Briefly, samples were retrieved from liquid nitrogen storage and aliquotted on ice under red light conditions before being refrozen and transported to the laboratory. Total lipids were extracted from plasma and separated by thin-layer chromatography into PPLs, triacylglycerol and cholesterol esters on silica gel plates (Silica gel $60 \mathrm{H}$ Merck Darmstadt Germany). PPL fatty acid methyl esters were separated and quantified with a Hewlett-Packard 5880 gas-liquid chromatograph using a capillary column equipped with flame ionization detection and Hewlett-Packard Chem-Station data system. The between batch coefficients of variation were between $1 \%$ and $12 \%$.

We measured the following \%PPL at baseline: total SFA; myristic acid (14:0); palmitic acid (16:0); stearic acid (18:0); total MUFA; palmitoleic acid (16:1n-7); oleic acid (18:1n-9); total PUFA; n-3 PUFA; $\alpha$-linolenic acid (ALA, 18:3n-3); EPA (20:5n-3); DHA (22:6n-3); docosapentaenoic (DPA, 22:5n-3); n-6 PUFA; linoleic acid (18:2n-6); dihomo- $\gamma$ linolenic acid (DGLA, 20:3n-6); arachidonic acid (20:4n-6); ratio of n-6 to n-3 PUFA (n-6:n-3 PUFA); and trans fatty acid (Trans, 16:1 18:1 and 18:2). For ten \%PPL fatty acid values with missing data (myristic acid, 3; palmitoleic acid, 1; and $\alpha$-linolenic acid, 6), we assumed that the levels were undetectable and assigned them a value of zero. 
Dietary intakes were estimated using a self-administered 121-item FFQ specifically developed for the MCCS and administered at baseline attendance ${ }^{15}$. Dietary energy intake (excluding alcohol) was calculated using sex-specific portion sizes and Australian food composition data. Dietary intakes of fatty acids were calculated using Australian fatty acid composition data ${ }^{16}$. To facilitate comparison of our results for \%PPL and dietary fatty acids we used the casecohort sample to investigate associations with dietary fatty acids.

\section{Assessment of other risk factors}

A structured interview schedule was used to collect information on potential risk factors: age, country of birth, alcohol consumption, leisure time physical activity, smoking status, educational attainment and family history of cancer.

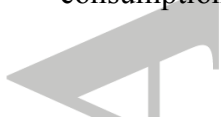

\section{Statistical Analysis}

Follow-up began at baseline attendance and continued until date of diagnosis of prostate cancer or unknown primary cancer, an unconfirmed diagnosis of prostate cancer, death, last known to be in Australia or 30 June 2002, whichever came first. Cox regression models were fitted, with age as the time axis, to estimate hazard ratios (HRs) and 95\% confidence intervals (CIs), separately for each \%PPL and dietary fatty acid. To allow for the case-cohort design, we used Prentice weights ${ }^{17}$ with robust standard errors. To test for heterogeneity in HRs by tumour aggressiveness, we fitted Cox regression models for competing risk using a data duplication method.

All models included country of birth (Southern Europe; Northern Europe, Australia or New Zealand); educational attainment; alcohol consumption (abstainers, former drinkers, 1-39 grams/day, 40-59 grams/day, $\geq 60$ grams/day); physical activity (an ordinal scale based on frequency and intensity of physical activity, then grouped based on approximate quartiles); daily non-alcohol energy intake and family history of cancer. Models were stratified by smoking status (never, former, current) as tests based on Schoenfeld residuals showed that the hazards were not proportional for this variable. Additional analyses involved fitting and testing two-way interaction terms between tumour aggressiveness and each confounder in the competing risk model: no interaction was statistically significant.

Intakes of energy and each dietary fatty acid were log transformed and then the dietary fatty acids were adjusted for energy intake using the residuals method ${ }^{18}$. Quintile cut points (Q) for each fatty acid were obtained from the subcohort, the lowest quintile (Q1) being the referent category. To estimate linear trends on a log hazard scale, each 
fatty acid was fitted as a pseudocontinuous covariate (using the median Q values). To investigate departures from linearity in the relationship between fatty acid intake and overall prostate cancer we also included a quadratic pseudocontinuous term for each fatty acid in these models, and assessed the significance of this term using the Wald test.

Differences in selected baseline characteristics and \%PPL and dietary fatty acids were assessed using chi-square tests for categorical variables and Wilcoxon rank-sum tests for continuous variables.

We also investigated whether further adjustment of our models for intakes (from food sources only) of lycopene ( $\mu \mathrm{g} / \mathrm{d}$ ), calcium $(\mathrm{mg} / \mathrm{d})$, and vitamin $\mathrm{E}(\mathrm{mg} / \mathrm{d})$ affected the estimates. We analysed the specific trans fatty acids separately but the estimates were similar to the total trans fatty acids so we have just presented these. Selected analyses were repeated after excluding the first 2 years of follow-up and excluding men who consumed fish-oil or cod-liver oil supplements (since we could not measure these accurately because our FFQ did not allow for a quantative assessment of these).

Statistical analyses were performed using Stata/SE 11.2 (Stata Corporation, College Station, TX). P $<0.05$ (two-sided) was considered statistically significant.

\section{Results}

Baseline characteristics are shown in Table 1. Cases were older, less likely to be born in Southern Europe, had lower physical activity levels, and were more likely to be current smokers and to have had a family history of cancer (all $\mathrm{P} \leq 0.01$ ). Median \%PPL fatty acid and daily energy-adjusted fatty acid intakes are shown in Table 2. Cases had significantly higher \%PPL SFA, trans fatty acids and dietary n-6:n-3 PUFA, and significantly lower \%PPL MUFA, oleic acid, ALA and dietary EPA and trans fatty acids (all $\mathrm{P} \leq 0.05)$.

Moderate Spearman correlations were observed between energy-adjusted dietary intakes and \%PPL levels for total PUFA $(\rho=0.28)$, n-3 PUFA $(\rho=0.21)$, DHA $(\rho=0.39)$, n-6 PUFA $(\rho=0.34)$, linoleic acid $(\rho=0.30)$ and n-6:n-3 PUFA $(\rho=0.39)$, whereas only weak correlations were observed for all other fatty acids (all $\rho<0.20)$. 
Table 3 shows HRs in relation to \%PPL fatty acids. We observed a positive association with total SFA (HR=1.51 [1.06, 2.16], (Q5 vs. Q1); P-trend=0.003), primarily due to palmitic acid for which HRs (Q2 to Q5) were elevated, although the test for linear trend was not significant. HRs less than unity were observed for myristic acid, but none was statistically significant. There was weak evidence of an inverse association with total MUFA (P-trend=0.07). A similar pattern was observed for oleic acid; where the HR for Q5 vs. Q1 was $0.62[0.43,0.91]$, P-trend=0.04. The inclusion of a quadratic fatty acid term indicated a departure from linearity $(\mathrm{P}$-quadratic trend $=0.001)$ for $\% \mathrm{PPL}$ palmitic acid, with a peak at Q3 $(\mathrm{HR}=2.16[1.46,3.20])$, but there was no evidence of a departure from linearity in the relationships for any other \%PPL fatty acid (all $\mathrm{P}>0.1)$.

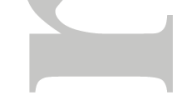

Table 4 shows HRs in relation to dietary intake of fatty acids. Although there were no statistically significant linear associations, HRs were all above unity for total PUFA and n-6 PUFA, largely attributable to linoleic acid intake. Elevated HRs (Q5 to Q2 vs. Q1) were observed for linoleic acid, but these were statistically significant only for Q2 and Q3. For n-6:n-3 PUFA, we observed an elevated HR for Q4 vs. Q1 but no other. The inclusion of a quadratic term suggested an inverted U-shaped relationship between total dietary PUFA, n-6 PUFA and linoleic acid and risk $(\mathrm{P}=0.03$, $\mathrm{P}=0.01$ and $\mathrm{P}=0.01$ respectively), but there was no evidence of departure from linearity for any other dietary fatty acid association (all $\mathrm{P}>0.1)$.

There were no associations between other \%PPL or dietary fatty acid estimates and prostate cancer incidence and no observed association varied significantly by tumour aggressiveness (test for homogeneity, all $\mathrm{P}>0.09$ ).

Further adjustment of the models for intakes of calcium, lycopene and vitamin E did not materially change the HR estimates, neither did the exclusion of the first two years of follow-up or those who consumed fish oil or cod-liver oil supplements.

Discussion

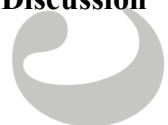

We report a positive linear association between \%PPL SFA and prostate cancer risk, a non-linear relationship (with a peak at Q3) for \%PPL palmitic acid and weak evidence of an inverse monotonic association for \%PPL MUFA, primarily due to oleic acid. Associations we observed for dietary fatty acid intakes were not consistent with those for \%PPL estimates. No association was observed to vary significantly by tumour aggressiveness. 
The case-cohort design is an efficient method for analysing time to failure in a large cohort where the outcome is rare, requiring exposure and covariate information on all failures and only a small subset of censored observations. Sampling in the case-cohort design is done a priori irrespective of case-status or time ${ }^{17}$. Other strengths of our study include virtually complete follow-up as the identification of incident prostate cancers was confirmed by record-linkage to cancer databases which have full geographic coverage. Our study limitations are common to others that use FFQs and biomarkers to estimate fatty acid intakes and composition. Intakes estimated from FFQs have considerable error ${ }^{19}$ that contributes to weak and inconsistent findings as reported in a recent meta-analysis of dietary fat and prostate cancer ${ }^{1}$. We measured \%PPL fatty acids, which reflect short-term rather than long-term intakes ${ }^{20}$. Further, correlations between fatty acids make it difficult to estimate associations that are specific to a single type. For subgroups, particularly aggressive tumours, small numbers led to low precision. Given the number of comparisons performed, some associations might be due to chance alone.

Due to difficulties with estimating dietary fat intakes, biomarkers are often used. However, SFAs can be synthesized endogenously from acetyl CoA and dietary saturated fatty acids can also be endogenously altered through elongation and desaturation to produce oleic acid ${ }^{21}$ thus PPL levels do not necessarily agree with intake estimates. Weaker correlations between \%PPL and dietary fatty acids for MUFA and some SFAs may also be attributed to the relatively small range of habitual intakes of these fatty acids and large day-to-day intra-individual variation. PUFAs cannot generally be produced endogenously, thus diet and supplements are the major source, which is reflected in the stronger correlations for these fatty acids. Fatty acid concentrations can also be affected by total amount of fat consumed, genetic, lifestyle and metabolic factors, which may affect de novo fatty acid synthesis and fatty acid metabolism ${ }^{21}$. In this and other studies, fatty acids concentrations are usually presented as a percentage of the total, reflecting relative concentrations. Thus, changes in the intake of one fatty acid might affect the relative concentration of others, particularly for those representing lower proportions. Given these limitations, it is not surprising that the associations with prostate cancer we observed were not consistent between dietary intakes and biomarkers.

A meta-analysis of 14 studies reported a weak positive association between dietary SFA and prostate cancer (but this association was not evident in studies that adjusted for energy intake) and stronger positive associations with advanced 
prostate cancer (based on 5 studies) but with substantial heterogeneity across studies ${ }^{1}$. A recent review concluded that the data were too limited to support an association between SFA intake and prostate cancer ${ }^{2}$. Our null finding for dietary SFA is consistent with several others ${ }^{22-28}$. Men consuming high SFA diets have been shown to have an increased risk of biochemical failure following prostatectomy, suggesting that SFAs might promote prostate cancer progression $^{29}$. A recent report on SFA and cardiovascular disease (CVD) noted that associations varied depending on the source of the SFA, with those from dairy having an inverse association while those from meat showed a positive association ${ }^{30}$. Although we do not have data to test whether the same holds for prostate cancer, we did not find any associations between dairy ${ }^{31}$ or meat intake and prostate cancer in our study. Fewer studies have investigated associations with biomarkers of total SFA and have not reported any association with prostate cancer ${ }^{32,33}$, in contrast to the positive association we observed.

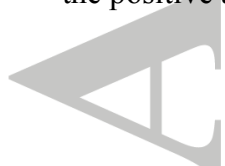

The increased prostate cancer risk we observed with higher levels of \%PPL palmitic acid is consistent with other studies of blood levels ${ }^{32,34}$ and intakes ${ }^{25}$. Other studies have generally reported no overall associations ${ }^{33,35,36}$ but some have found positive associations for specific subgroups ${ }^{33,36}$.

Palm oil, butter, milk, cheese and meat are the main sources of palmitic acid, which can be synthesised from other fatty acids and is the major fatty acid produced by de novo lipogenesis from acetyl CoA and malonyl CoA by the enzyme fatty acid synthase. There is some evidence that de novo fatty acids are required for prostate cancer cell proliferation and survival ${ }^{37}$ but it is still unknown whether prostate cancer cells differentially utilise de novo and dietary lipids ${ }^{37}$. The increased risks we observed for palmitic acid might reflect higher fatty acid synthase activity.

Consistent with others, we observed no association with \%PPL myristic ${ }^{23,34,35}$ or stearic acid ${ }^{23,32,33,36}$ while other studies have reported positive associations with circulating myristic acid ${ }^{32,36}$, inverse associations with circulating stearic acid ${ }^{34,35}$ and positive associations with myristic acid intake ${ }^{25}$.

Consistent with our null finding for total dietary MUFA intake, a meta-analysis of 9 studies as well as most subsequent studies found no association with either intake ${ }^{1,22,24,26,28,38}$ or blood levels ${ }^{32,33}$ of total MUFA. Oleic acid is the main 
MUFA in both the diet and circulation. Studies of circulating oleic acid concentrations have generally shown no association with prostate cancer ${ }^{32-36}$, in contrast to the inverse association observed by us and one other study ${ }^{23}$. This could relate to different sources of oleic acid in the circulation. Olive oil is a particularly rich source of oleic acid but also contains other molecules which may contribute to health benefits ${ }^{39}$. In Australia, red meat (an important source of MUFA) is the main source of oleic acid ${ }^{40}$, but this does not provide antioxidants. Several molecular mechanisms have been proposed to underlie a protective effect of oleic acid, including hormonal status, cell membrane structure and function, signal transduction pathways, gene expression and the immune system ${ }^{41}$. Our finding of no association between dietary oleic acid and prostate cancer is consistent with a meta-analysis including 3 case-control studies, which reported no association for olive oil or MUFA derived from this ${ }^{39}$. The null association we observed for both dietary intake and \%PPL of palmitoleic acid is consistent with others ${ }^{33,34,36}$.

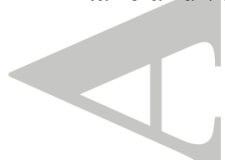

Consistent with our null finding, two meta-analyses of prospective epidemiological studies report no association with $\alpha$ linolenic acid (biomarker and intakes combined) and prostate cancer risk ${ }^{4,42}$. In contrast, another meta-analysis of prospective studies reported slightly reduced risks of prostate cancer with dietary intake of $\alpha$-linolenic acid ${ }^{43}$ whereas a systematic review suggested that higher intakes might increase risk ${ }^{3}$. The European Prospective Investigation into Cancer and Nutrition study, not included in these reviews, also reported a positive association for \%PPL $\alpha$-linolenic acid with high-grade prostate cancer only ${ }^{34}$ and a recent case-control study reported an increased risk of total prostate cancer with high dietary intake but no association with blood levels ${ }^{23}$. Inconsistencies between biomarkers and dietary intakes for $\alpha$-linolenic acid likely reflect that the levels in plasma phospholipids are very low and the correlations between diet and PPL\% are low relative to other PUFA, as we showed previously for the MCCS ${ }^{14}$.

Although, we did not observe any clear associations between \%PPL or dietary EPA or DHA and prostate cancer, all HRs were below unity for EPA and DHA intakes. Meta-analyses of epidemiological studies ${ }^{4,7}$ and recent studies of circulating long chain n-3 fatty acids $33,34,44,45$, report no clear associations with EPA or DHA; there is also no strong evidence for a protective effect of fish consumption ${ }^{6,7}$, the main dietary source of these. Evidence from experimental studies suggests that n-3 PUFA, especially long-chain n-3 PUFA such as EPA and DHA, are negatively associated with prostate cancer $^{3,46}$ whereas n-6 PUFA stimulates cancer progression ${ }^{11}$ and several mechanisms have been proposed ${ }^{8,12}$. 
Studies of n-6 PUFAs have mainly focused on linoleic acid. We observed a threshold effect for dietary linoleic acid, with the highest HRs for the $2^{\text {nd }}$ and $3^{\text {rd }}$ quintiles relative to the lowest, but this finding was not supported by our results for $\%$ PPL, despite the moderate correlation between dietary and \%PPL estimates and the fact that linoleic acid can only be acquired from diet. Others have generally failed to find clear evidence of an association with linoleic acid ${ }^{3,9,33,34,44}$ but an inverse association was reported by the Physicians Health Study ${ }^{45}$. On the other hand, our findings for dietary linoleic acid are consistent with animal and in vitro studies that have shown it to promote prostate cancer growth ${ }^{11}$. Several complex metabolic processes have been postulated to explain this association ${ }^{47}$.

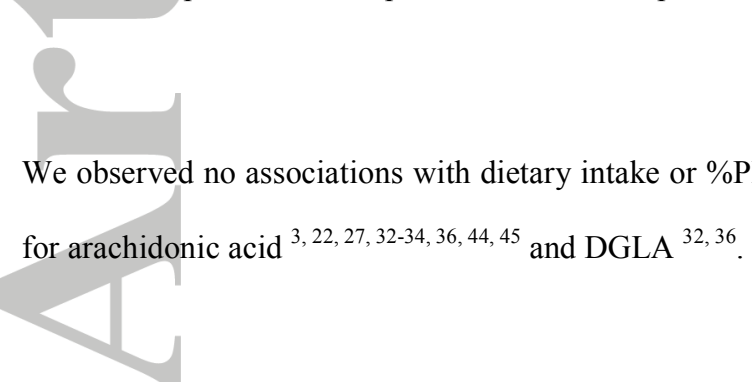

Several reasons have been proposed for the discrepancies between results from epidemiological and experimental studies in relation to long chain n-3 PUFAs ${ }^{12}$, including: intake of these PUFAs or within-population variability might be too low in some populations to show a protective effect; and n-3 PUFAs are usually analysed without taking into account n-6 PUFAs. The n-3 PUFAs compete with n-6 PUFAs for metabolism into eicosanoids, with those derived from n-6 PUFAs tending to be inflammatory and possibly promoting carcinogenesis ${ }^{21}$. We examined the association of n-6:n-3 PUFA but did not find an association for dietary intake or \%PPL. The level of exposure to fatty acids in animal studies tends to be much higher compared with humans and long chain n-3 PUFAs can be biosynthesized from $\alpha$ linolenic acid more actively in animals than humans ${ }^{3,12}$, similarly with the biosynthesis of arachidonic acid (a precursor of eicosanoids) from linoleic acid ${ }^{3}$.

We did not observe any association between either \%PPL or dietary intake of trans fatty acids, which is consistent with two studies of dietary intakes ${ }^{22,27}$ but inconsistent with positive associations reported by others ${ }^{38,48,49}$ and the inverse association for serum trans fatty acid 18:1 and 18:2 and high-grade prostate cancer reported by the Prostate Cancer Prevention Trial ${ }^{44}$. These mainly North American populations ${ }^{38,44,48,49}$ are likely to have higher average trans fatty intakes and plasma levels than Australia ${ }^{50}$; this might partly explain why we did not observe any associations with trans fatty acids. 
While some studies have shown different associations by grade or aggressiveness of tumour $24,34,44,45$, suggesting that some fatty acids might differentially affect initiation and progression, few of these formally tested for heterogeneity.

None of our observed associations differed significantly by tumour aggressiveness.

Our findings suggest risk of prostate cancer is positively associated with \%PPL SFA, primarily with palmitic acid and reak weak inversely associated with \%PPL MUFA, primarily with oleic acid. Higher risks were also observed for dietary n-6 PUFA, primarily with linoleic acid. However, given the weakness of these associations and the inconsistent results between studies, there is little evidence that dietary fatty acid composition is an important contributor to prostate cancer

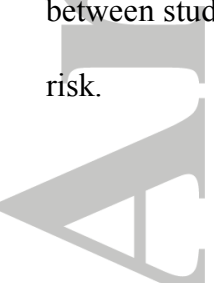

\section{Acknowledgements}

Cohort recruitment was funded by VicHealth and The Cancer Council Victoria. This work was supported by infrastructure from the Cancer Council Victoria and grants from the National Health and Medical Research Council $(209057,251533,520316)$. This study was made possible by the contribution of many people, including the original investigators and the diligent team who recruited the participants and who continue working on follow-up. We would also like to express our gratitude to the many thousands of Melbourne residents who continue to participate in the study. We would like to acknowledge the work of Amalia Karahalios who checked all statistical analyses.

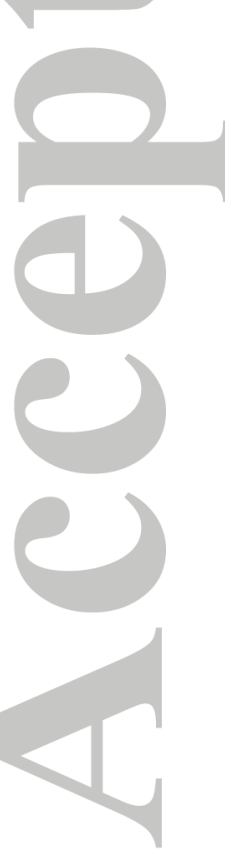


1. Dennis LK, Snetselaar LG, Smith BJ, Stewart RE, Robbins ME. Problems with the assessment of dietary fat in prostate cancer studies. Am J Epidemiol 2004;160:436-44.

2. Gerber M. Background review paper on total fat, fatty acid intake and cancers. Ann Nutr Metab $2009 ; 55: 140-61$.

3. Astorg P. Dietary N-6 and N-3 polyunsaturated fatty acids and prostate cancer risk: a review of epidemiological and experimental evidence. Cancer Causes Control 2004;15:367-86.

4. Brouwer IA. Omega-3 PUFA: good or bad for prostate cancer? Prostaglandins Leukot Essent Fatty Acids 2008;79:97-9.

5. Gerber M. Omega-3 fatty acids and cancers: a systematic update review of epidemiological studies. Br J Nutr 2012;107 Suppl 2:S228-39.

6. Szymanski KM, Wheeler DC, Mucci LA. Fish consumption and prostate cancer risk: a review and metaanalysis. Am J Clin Nutr 2010;92:1223-33.

7. Terry PD, Rohan TE, Wolk A. Intakes of fish and marine fatty acids and the risks of cancers of the breast and prostate and of other hormone-related cancers: a review of the epidemiologic evidence. Am J Clin Nutr $2003 ; 77: 532-43$.

8. Terry PD, Terry JB, Rohan TE. Long-chain (n-3) fatty acid intake and risk of cancers of the breast and the prostate: recent epidemiological studies, biological mechanisms, and directions for future research. J Nutr 2004; 134:3412S-20S.

9. Zock PL, Katan MB. Linoleic acid intake and cancer risk: a review and meta-analysis. Am J Clin Nutr $1998 ; 68: 142-53$.

10. Brouwer IA, Katan MB, Zock PL. Dietary alpha-linolenic acid is associated with reduced risk of fatal coronary heart disease, but increased prostate cancer risk: a meta-analysis. J Nutr 2004;134:919-22.

11. Rose DP. Effects of dietary fatty acids on breast and prostate cancers: evidence from in vitro experiments and animal studies. Am J Clin Nutr 1997;66:1513S-22S.

12. Larsson SC, Kumlin M, Ingelman-Sundberg M, Wolk A. Dietary long-chain n-3 fatty acids for the prevention of cancer: a review of potential mechanisms. Am J Clin Nutr 2004;79:935-45.

13. Giles GG, English DR. The Melbourne Collaborative Cohort Study. IARC Sci Publ 2002;156:69-70. 
14. Hodge AM, Simpson JA, Gibson RA, Sinclair AJ, Makrides M, O'Dea K, English DR, Giles GG. Plasma phospholipid fatty acid composition as a biomarker of habitual dietary fat intake in an ethnically diverse cohort. Nutr Metab Cardiovasc Dis 2007; 17:415-26.

15. Ireland P, Jolley D, Giles G, O’Dea K, Powles J, Rutishauser I, Wahlqvist ML, Williams J. Development of the Melbourne FFQ: a food frequency questionnaire for use in an Australian prospective study involving an ethnically diverse cohort. Asia Pac J Clin Nutr 1994;3:19-31.

16. RMIT Lipid Research Group. Fatty acid compositional database Brisbane: Xyris software, 2001.

17. Prentice RL. A case-cohort design for epidemiologic cohort studies and disease prevention trials. Biometrika 1986;73:1-11.

18. Willett W, Stampfer MJ. Total energy intake: implications for epidemiologic analyses. Am J Epidemiol $1986 ; 124: 17-27$

19. Jacobs DR, Jr., Anderson JT, Blackburn H. Diet and serum cholesterol: do zero correlations negate the relationship? Am J Epidemiol 1979;110:77-87.

20. Arab L. Biomarkers of fat and fatty acid intake. J Nutr 2003;133 Suppl 3:925S-32S.

21. Food and Agriculture Organization of the United Nations. Fats and fatty acids in human nutrition: Report of an expert consultation. FAO Food and Nutrition Paper 91, 2010.

22. Hodge AM, English DR, McCredie MR, Severi G, Boyle P, Hopper JL, Giles GG. Foods, nutrients and prostate cancer. Cancer Causes Control 2004;15:11-20.

23. Jackson MD, Walker SP, Simpson-Smith CM, Lindsay CM, Smith G, McFarlane-Anderson N, Bennett FI, Coard KC, Aiken WD, Tulloch T, Paul TJ, Wan RL. Associations of whole-blood fatty acids and dietary intakes with prostate cancer in Jamaica. Cancer Causes Control 2012;23:23-33.

24. Kristal AR, Arnold KB, Neuhouser ML, Goodman P, Platz EA, Albanes D, Thompson IM. Diet, supplement use, and prostate cancer risk: results from the prostate cancer prevention trial. Am J Epidemiol 2010;172:566-77.

25. Kurahashi N, Inoue M, Iwasaki M, Sasazuki S, Tsugane AS. Dairy product, saturated fatty acid, and calcium intake and prostate cancer in a prospective cohort of Japanese men. Cancer Epidemiol Biomarkers Prev 2008;17:930-7.

26. Park SY, Murphy SP, Wilkens LR, Henderson BE, Kolonel LN. Fat and meat intake and prostate cancer risk: the multiethnic cohort study. Int J Cancer 2007;121:1339-45. 
27. Schuurman AG, van den Brandt PA, Dorant E, Brants HA, Goldbohm RA. Association of energy and fat intake with prostate carcinoma risk: results from The Netherlands Cohort Study. Cancer 1999;86:1019-27.

28. Crowe FL, Key TJ, Appleby PN, Travis RC, Overvad K, Jakobsen MU, Johnsen NF, Tjonneland A, Linseisen J, Rohrmann S, Boeing H, Pischon T, et al. Dietary fat intake and risk of prostate cancer in the European Prospective Investigation into Cancer and Nutrition. Am J Clin Nutr 2008;87:1405-13.

29. Strom SS, Yamamura Y, Forman MR, Pettaway CA, Barrera SL, DiGiovanni J. Saturated fat intake predicts biochemical failure after prostatectomy. Int J Cancer 2008;122:2581-5.

30. de Oliveira Otto MC, Mozaffarian D, Kromhout D, Bertoni AG, Sibley CT, Jacobs DR, Jr., Nettleton JA. Dietary intake of saturated fat by food source and incident cardiovascular disease: the Multi-Ethnic Study of Atherosclerosis. Am J Clin Nutr 2012;96:397-404.

31. Severi G, English DR, Hopper JL, Giles GG. Re: Prospective studies of dairy product and calcium intakes and prostate cancer risk: a meta-analysis. J Natl Cancer Inst 2006;98:794-5; author reply 95.

32. Harvei S, Bjerve KS, Tretli S, Jellum E, Robsahm TE, Vatten L. Prediagnostic level of fatty acids in serum phospholipids: omega-3 and omega-6 fatty acids and the risk of prostate cancer. Int J Cancer 1997;71:545-51.

33. Park SY, Wilkens LR, Henning SM, Le Marchand L, Gao K, Goodman MT, Murphy SP, Henderson BE, Kolonel LN. Circulating fatty acids and prostate cancer risk in a nested case-control study: the Multiethnic Cohort. Cancer Causes Control 2009;20:211-23.

34. Crowe FL, Allen NE, Appleby PN, Overvad K, Aardestrup IV, Johnsen NF, Tjonneland A, Linseisen J, Kaaks R, Boeing H, Kroger J, Trichopoulou A, et al. Fatty acid composition of plasma phospholipids and risk of prostate cancer in a case-control analysis nested within the European Prospective Investigation into Cancer and Nutrition. Am J Clin Nutr 2008;88:1353-63.

35. Gann PH, Hennekens CH, Sacks FM, Grodstein F, Giovannucci EL, Stampfer MJ. Prospective study of plasma fatty acids and risk of prostate cancer. J Natl Cancer Inst 1994;86:281-6.

36. Mannisto S, Pietinen P, Virtanen MJ, Salminen I, Albanes D, Giovannucci E, Virtamo J. Fatty acids and risk of prostate cancer in a nested case-control study in male smokers. Cancer Epidemiol Biomarkers Prev $2003 ; 12: 1422-8$

37. Suburu J, Chen YQ. Lipids and prostate cancer. Prostaglandins Other Lipid Mediat 2012;98:1-10.

38. Hu J, La Vecchia C, Gibbons L, Negri E, Mery L. Nutrients and risk of prostate cancer. Nutr Cancer $2010 ; 62: 710-8$. 
39. Psaltopoulou T, Kosti RI, Haidopoulos D, Dimopoulos M, Panagiotakos DB. Olive oil intake is inversely related to cancer prevalence: a systematic review and a meta-analysis of 13,800 patients and 23,340 controls in 19 observational studies. Lipids Health Dis 2011;10:127.

40. Baghurst K, Record S, Leppard P. Red meat consumption in Australia: intakes, nutrient contribution and changes over time. Aust J Nutr Diet 2000;57:3S-36S.

41. Escrich E, Moral R, Grau L, Costa I, Solanas M. Molecular mechanisms of the effects of olive oil and other dietary lipids on cancer. Mol Nutr Food Res 2007;51:1279-92.

42. Simon JA, Chen $\mathrm{YH}$, Bent S. The relation of alpha-linolenic acid to the risk of prostate cancer: a systematic review and meta-analysis. Am J Clin Nutr 2009;89:1558S-64S.

43. Chua ME, Sio MC, Sorongon MC, Dy JS. Relationship of dietary intake of omega-3 and omega-6 Fatty acids with risk of prostate cancer development: a meta-analysis of prospective studies and review of literature. Prostate Cancer 2012;2012:826254.

44. Brasky TM, Till C, White E, Neuhouser ML, Song X, Goodman P, Thompson IM, King IB, Albanes D, Kristal AR. Serum phospholipid fatty acids and prostate cancer risk: results from the prostate cancer prevention trial. Am J Epidemiol 2011;173:1429-39.

45. Chavarro JE, Stampfer MJ, Li H, Campos H, Kurth T, Ma J. A prospective study of polyunsaturated fatty acid levels in blood and prostate cancer risk. Cancer Epidemiol Biomarkers Prev 2007;16:1364-70.

46. Rose DP. Dietary fatty acids and prevention of hormone-responsive cancer. Proc Soc Exp Biol Med $1997 ; 216: 224-33$

47. Berquin IM, Min Y, Wu R, Wu J, Perry D, Cline JM, Thomas MJ, Thornburg T, Kulik G, Smith A, Edwards IJ, D'Agostino R, et al. Modulation of prostate cancer genetic risk by omega-3 and omega-6 fatty acids. J Clin Invest 2007; 117:1866-75.

48. Chavarro JE, Stampfer MJ, Campos H, Kurth T, Willett WC, Ma J. A prospective study of trans-fatty acid levels in blood and risk of prostate cancer. Cancer Epidemiol Biomarkers Prev 2008;17:95-101.

49. Liu X, Schumacher FR, Plummer SJ, Jorgenson E, Casey G, Witte JS. Trans-fatty acid intake and increased risk of advanced prostate cancer: modification by RNASEL R462Q variant. Carcinogenesis 2007;28:1232-6.

50. Craig-Schmidt MC. World-wide consumption of trans fatty acids. Atheroscler Suppl 2006;7:1-4. 
Table 1. Baseline characteristics of prostate cancer cases and non-cases

\begin{tabular}{|c|c|c|c|c|c|}
\hline 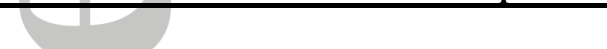 & \multicolumn{2}{|c|}{ Non-cases } & \multicolumn{2}{|r|}{ Cases } & \multirow[t]{2}{*}{ P-value ${ }^{a}$} \\
\hline No. of participants & 1661 & & 464 & & \\
\hline Age at baseline, Median $\left(25^{\text {th }}-75^{\text {th }}\right.$ percentile $)$ & 54.2 & $(46.4-61.7)$ & 63.2 & $(58.5-66.6)$ & $<0.0001$ \\
\hline Energy $^{\mathrm{b}}(\mathrm{kJ} / \mathrm{d})$, Median $\left(25^{\text {th }}-75^{\text {th }}\right.$ percentile $)$ & 9007 & $(7346-11463)$ & 9204 & $(7211-11214)$ & 0.71 \\
\hline \multicolumn{6}{|l|}{ Country of birth, $n(\%)$} \\
\hline Southern Europe & 465 & $(28)$ & 93 & $(20)$ & \\
\hline Northern Europe, Australia or New Zealand & 1196 & $(72)$ & 371 & $(80)$ & 0.001 \\
\hline \multicolumn{6}{|l|}{ Education, $n(\%)$} \\
\hline$\leq$ primary school & 287 & $(17)$ & 74 & $(16)$ & \\
\hline some high/technical school & 487 & (29) & 158 & (34) & \\
\hline completed high/technical school & 420 & $(25)$ & 126 & (27) & \\
\hline degree/diploma & 467 & (28) & 106 & (23) & 0.06 \\
\hline \multicolumn{6}{|l|}{ Alcohol consumption, $n(\%)$} \\
\hline Abstainers & 213 & (13) & 75 & $(16)$ & \\
\hline Ex-drinkers & 70 & (4) & 24 & (5) & \\
\hline Low intake $(1-39 \mathrm{~g} / \mathrm{d})$ & 1087 & $(65)$ & 291 & (63) & \\
\hline Medium intake $(40-59 \mathrm{~g} / \mathrm{d})$ & 169 & $(10)$ & 42 & (9) & \\
\hline High intake $(\geq 60 \mathrm{~g} / \mathrm{d})$ & 122 & (7) & 32 & (7) & 0.32 \\
\hline \multicolumn{6}{|l|}{ Physical activity score, $n(\%)$} \\
\hline 0 & 364 & $(22)$ & 91 & $(20)$ & \\
\hline$>0$ to $<4$ & 340 & $(20)$ & 95 & (20) & \\
\hline 4 to $<6$ & 531 & (32) & 183 & (39) & \\
\hline$\geq 6$ & 426 & (26) & 95 & (20) & 0.01 \\
\hline \multicolumn{6}{|l|}{ Smoking status, $n(\%)$} \\
\hline Never & 708 & (43) & 192 & $(41)$ & \\
\hline Former & 267 & (16) & 52 & (11) & \\
\hline Current & 686 & (41) & 220 & (47) & 0.01 \\
\hline \multicolumn{6}{|l|}{ Family history of cancer, $n(\%)$} \\
\hline No & 1113 & $(67)$ & 275 & $(59)$ & \\
\hline Yes & 548 & (33) & 189 & (41) & 0.002 \\
\hline
\end{tabular}

NOTE: percentages may not add up to $100 \%$ due to rounding.

${ }^{\mathrm{a}} \mathrm{P}$-value from chi-squared test for categorical variables and from Wilcoxon rank-sum test for continuous variables

${ }^{\mathbf{b}}$ Energy from food sources only (excludes energy from alcohol) 
Table 2. Median $\left(25^{\text {th }}-75^{\text {th }}\right.$ percentile $) \%$ PPL and dietary fatty acids for prostate cancer cases and non-cases

\begin{tabular}{|c|c|c|c|c|c|}
\hline 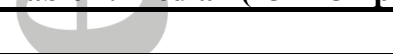 & \multicolumn{2}{|c|}{ Non-cases } & \multicolumn{2}{|r|}{ Cases } & \multirow[t]{2}{*}{ P-value ${ }^{\mathbf{a}}$} \\
\hline$\%$ PPL Fatty Acids (mass \% & & & & & \\
\hline Total SFA & 43.2 & $(42.5-43.9)$ & 43.4 & $(42.7-44.0)$ & 0.02 \\
\hline Myristic acid (14:0) & 0.23 & $(0.19-0.27)$ & 0.23 & $(0.18-0.28)$ & 0.59 \\
\hline Palmitic acid (16:0) & 25.4 & $(24.4-26.3)$ & 25.4 & $(24.6-26.3)$ & 0.13 \\
\hline Stearic acid (18:0) & 14.4 & $(13.6-15.1)$ & 14.4 & $(13.7-15.1)$ & 0.92 \\
\hline Total MUFA & 13.0 & $(11.9-14.2)$ & 12.7 & $(11.5-14.1)$ & 0.01 \\
\hline Palmitoleic acid (16:1n-7) & 0.36 & $(0.28-0.48)$ & 0.35 & $(0.28-0.47)$ & 0.64 \\
\hline Oleic acid (18:1n-9) & 9.6 & $(8.68-10.63)$ & 9.3 & $(8.38-10.46)$ & 0.002 \\
\hline Total PUFA & 42.5 & $(41.2-43.7)$ & 42.6 & $(41.2-43.8)$ & 0.75 \\
\hline n-3 PUFA & 6.44 & $(5.72-7.37)$ & 6.53 & $(5.74-7.41)$ & 0.45 \\
\hline $\operatorname{ALA}(18: 3 n-3)$ & 0.14 & $(0.11-0.20)$ & 0.13 & $(0.10-0.19)$ & 0.03 \\
\hline EPA $(20: 5 n-3)$ & 0.97 & $(0.75-1.31)$ & 0.98 & $(0.71-1.28)$ & 0.37 \\
\hline DHA $(22: 6 n-3)$ & 3.82 & $(3.22-4.54)$ & 3.91 & $(3.26-4.59)$ & 0.27 \\
\hline DPA $(22: 5 n-3)$ & 1.26 & $(1.11-1.43)$ & 1.25 & $(1.10-1.42)$ & 0.996 \\
\hline n-6 PUFA & 35.5 & $(33.8-37.1)$ & 35.5 & $(33.6-37.1)$ & 0.86 \\
\hline Linoleic acid (18:2n-6) & 20.0 & $(18.1-22.0)$ & 19.9 & $(18.0-22.1)$ & 0.90 \\
\hline DGLA (20:3n-6) & 3.47 & $(3.01-4.03)$ & 3.41 & $(2.96-3.96)$ & 0.26 \\
\hline Arachidonic acid $(20: 4 n-6)$ & 10.4 & $(9.1-11.6)$ & 10.4 & $(9.1-11.8)$ & 0.87 \\
\hline n-6:n-3 PUFA & 5.46 & $(4.70-6.34)$ & 5.46 & $(4.58-6.35)$ & 0.59 \\
\hline Trans & 0.91 & $(0.70-1.15)$ & 1.00 & $(0.75-1.22)$ & $<0.0001$ \\
\hline \multicolumn{6}{|c|}{ Dietary Fatty Acids ${ }^{\text {b }}$ (grams/day) } \\
\hline Total SFA & 34.2 & $(29.3-39.5)$ & 34.6 & $(29.5-40.0)$ & 0.45 \\
\hline Myristic acid (14:0) & 3.24 & $(2.67-3.89)$ & 3.33 & $(2.66-4.03)$ & 0.44 \\
\hline Palmitic acid (16:0) & 18.1 & $(15.6-20.5)$ & 18.0 & $(15.7-20.5)$ & 0.90 \\
\hline Stearic acid (18:0) & 8.60 & $(7.24-10.01)$ & 8.61 & $(7.20-10.11)$ & 0.81 \\
\hline Total MUFA & 31.8 & $(27.7-35.8)$ & 31.3 & $(27.5-35.3)$ & 0.22 \\
\hline Palmitoleic acid (16:1n-7) & 1.94 & $(1.62-2.30)$ & 1.90 & $(1.60-2.22)$ & 0.11 \\
\hline Oleic acid (18:1n-9) & 29.2 & $(25.3-32.8)$ & 28.7 & $(25.2-32.3)$ & 0.23 \\
\hline Total PUFA & 13.3 & $(10.8-16.0)$ & 13.2 & $(11.2-15.8)$ & 0.67 \\
\hline n-3 PUFA & 1.32 & $(1.15-1.52)$ & 1.29 & $(1.13-1.49)$ & 0.06 \\
\hline ALA (18:3n-3) & 1.04 & $(0.90-1.17)$ & 1.02 & $(0.90-1.14)$ & 0.11 \\
\hline EPA $(20: 5 n-3)$ & 0.085 & $(0.059-0.119)$ & 0.081 & $(0.053-0.110)$ & 0.03 \\
\hline DHA (22:6n-3) & 0.153 & $(0.106-0.207)$ & 0.146 & $(0.099-0.192)$ & 0.09 \\
\hline DPA $(22: 5 n-3)$ & 0.026 & $(0.017-0.037)$ & 0.025 & $(0.015-0.036)$ & 0.24 \\
\hline n-6 PUFA & 11.9 & $(9.5-14.4)$ & 11.7 & $(10.0-14.4)$ & 0.54 \\
\hline Linoleic acid (18:2n-6) & 11.8 & $(9.4-14.3)$ & 11.6 & $(9.9-14.4)$ & 0.54 \\
\hline DGLA (20:3n-6) & 0.018 & $(0.011-0.030)$ & 0.018 & $(0.011-0.031)$ & 0.79 \\
\hline Arachidonic acid (20:4n-6) & 0.040 & $(0.029-0.054)$ & 0.039 & $(0.029-0.054)$ & 0.93 \\
\hline n-6:n-3 PUFA & 8.78 & $(7.26-10.80)$ & 9.19 & $(7.46-11.14)$ & 0.03 \\
\hline Trans & 0.105 & $(0.041-0.170)$ & 0.095 & $(0.036-0.167)$ & 0.05 \\
\hline
\end{tabular}

${ }^{\mathrm{a}} \mathrm{P}$-value from Wilcoxon rank-sum test for continuous variables

${ }^{\mathrm{b}}$ Adjusted for energy. 
Table 3. Hazard ratios ${ }^{\mathrm{a}}$ (HRs) and $95 \%$ confidence intervals (CIs) for prostate cancer risk associated with \%PPL fatty acids (quintiles)

\begin{tabular}{|c|c|c|c|c|c|c|c|c|c|c|c|c|}
\hline & \multicolumn{2}{|c|}{ Q1 } & \multicolumn{2}{|r|}{ Q2 } & \multicolumn{2}{|r|}{ Q3 } & \multicolumn{2}{|r|}{ Q4 } & \multicolumn{2}{|r|}{ Q5 } & \multirow{2}{*}{$\begin{array}{l}\text { Linear model } \\
\text { HR }(95 \% \text { CI })\end{array}$} & \multirow[t]{2}{*}{ P-trend } \\
\hline & Cases & HR & Cases & HR $(95 \%$ CI) & Cases & HR $(95 \%$ CI) & Cases & HR $(95 \%$ CI $)$ & Cases & HR (95\% CI) & & \\
\hline SFA & 78 & 1.00 & 72 & $1.01(0.69,1.48)$ & 107 & $1.48(1.04,2.11)$ & 103 & $1.57(1.10,2.25)$ & 104 & $1.51(1.06,2.16)$ & $1.18(1.06,1.32)$ & 0.003 \\
\hline Myristic acid (14:0) & 117 & 1.00 & 42 & $0.68(0.45,1.04)$ & 91 & $0.83(0.59,1.16)$ & 119 & $0.83(0.60,1.13)$ & 95 & $0.95(0.68,1.35)$ & $0.99(0.88,1.12)$ & 0.92 \\
\hline Palmitic acid (16:0) & 59 & 1.00 & 102 & $1.97(1.33,2.90)$ & 119 & $2.16(1.46,3.20)$ & 92 & $1.64(1.10,2.45)$ & 92 & $1.63(1.10,2.44)$ & $1.09(0.98,1.22)$ & 0.11 \\
\hline Stearic acid (18:0) & 89 & 1.00 & 92 & $1.08(0.76,1.53)$ & 105 & $1.15(0.81,1.65)$ & 90 & $1.19(0.83,1.70)$ & 88 & $1.03(0.72,1.48)$ & $1.02(0.91,1.14)$ & 0.74 \\
\hline MUFA & 131 & 1.00 & 85 & $0.78(0.55,1.09)$ & 74 & $0.69(0.48,0.98)$ & 94 & $0.85(0.60,1.20)$ & 80 & $0.67(0.46,0.98)$ & $0.89(0.79,1.01)$ & 0.07 \\
\hline Palmitoleic acid (16:1n-7) & 106 & 1.00 & 97 & $1.06(0.75,1.49)$ & 91 & $1.05(0.74,1.50)$ & 83 & $1.00(0.69,1.44)$ & 87 & $0.84(0.59,1.19)$ & $0.93(0.83,1.05)$ & 0.25 \\
\hline Oleic acid (18:1n-9) & 121 & 1.00 & 94 & $0.91(0.65,1.28)$ & 83 & $0.85(0.60,1.21)$ & 98 & $0.99(0.70,1.40)$ & 68 & $0.62(0.43,0.91)$ & $0.88(0.78,0.99)$ & 0.04 \\
\hline PUFA & 85 & 1.00 & 102 & $1.27(0.90,1.81)$ & 91 & $1.17(0.82,1.67)$ & 89 & $1.04(0.72,1.50)$ & 97 & $1.10(0.76,1.59)$ & $1.00(0.90,1.13)$ & 0.94 \\
\hline n-3 PUFA & 90 & 1.00 & 90 & $0.86(0.60,1.23)$ & 87 & $0.93(0.64,1.33)$ & 96 & $1.04(0.73,1.48)$ & 101 & $1.08(0.76,1.54)$ & $1.05(0.94,1.18)$ & 0.38 \\
\hline $\operatorname{ALA}(18: 3 n-3)$ & 116 & 1.00 & 82 & $1.06(0.74,1.50)$ & 89 & $1.04(0.74,1.46)$ & 94 & $1.02(0.73,1.42)$ & 83 & $0.90(0.63,1.28)$ & $0.96(0.86,1.08)$ & 0.50 \\
\hline EPA $(20: 5 n-3)$ & 111 & 1.00 & 73 & $0.71(0.50,1.02)$ & 109 & $1.19(0.85,1.66)$ & 75 & $0.91(0.63,1.31)$ & 96 & $1.06(0.75,1.51)$ & $1.05(0.93,1.18)$ & 0.42 \\
\hline DHA (22:6n-3) & 85 & 1.00 & 93 & $0.95(0.66,1.37)$ & 91 & $0.88(0.61,1.26)$ & 104 & $1.12(0.78,1.60)$ & 91 & $0.90(0.63,1.31)$ & $0.99(0.88,1.11)$ & 0.86 \\
\hline DPA $(22: 5 n-3)$ & 98 & 1.00 & 89 & $0.94(0.66,1.33)$ & 76 & $1.05(0.72,1.52)$ & 116 & $1.12(0.80,1.57)$ & 85 & $0.91(0.63,1.30)$ & $0.99(0.89,1.11)$ & 0.89 \\
\hline n-6 PUFA & 101 & 1.00 & 82 & $0.82(0.58,1.17)$ & 88 & $0.97(0.68,1.39)$ & 110 & $1.05(0.74,1.48)$ & 83 & $0.76(0.53,1.09)$ & $0.95(0.85,1.06)$ & 0.37 \\
\hline Linoleic acid (18:2n-6) & 95 & 1.00 & 94 & $1.07(0.75,1.53)$ & 100 & $1.14(0.80,1.63)$ & 76 & $0.72(0.50,1.04)$ & 99 & $0.98(0.68,1.40)$ & $0.95(0.85,1.07)$ & 0.41 \\
\hline DGLA (20:3n-6) & 100 & 1.00 & 105 & $1.13(0.80,1.59)$ & 76 & $0.79(0.55,1.12)$ & 98 & $1.18(0.83,1.67)$ & 85 & $0.96(0.68,1.36)$ & $0.99(0.89,1.11)$ & 0.89 \\
\hline Arachidonic acid (20:4n-6) & 101 & 1.00 & 83 & $0.91(0.64,1.31)$ & 89 & $1.10(0.77,1.56)$ & 89 & $0.99(0.70,1.42)$ & 102 & $1.15(0.81,1.62)$ & $1.05(0.94,1.18)$ & 0.39 \\
\hline n-6:n-3 PUFA & 99 & 1.00 & 89 & $1.03(0.72,1.48)$ & 94 & $0.81(0.57,1.15)$ & 84 & $0.94(0.64,1.37)$ & 98 & $0.99(0.69,1.42)$ & $1.00(0.89,1.13)$ & 0.99 \\
\hline Trans & 75 & 1.00 & 79 & $0.97(0.65,1.45)$ & 75 & $0.84(0.55,1.27)$ & 126 & $1.13(0.76,1.69)$ & 109 & $0.83(0.54,1.26)$ & $0.96(0.84,1.09)$ & 0.49 \\
\hline
\end{tabular}

Models adjusted for: country of birth, education, alcohol intake, physical activity, total energy intake from food and family history of cancer and stratified by smoking status

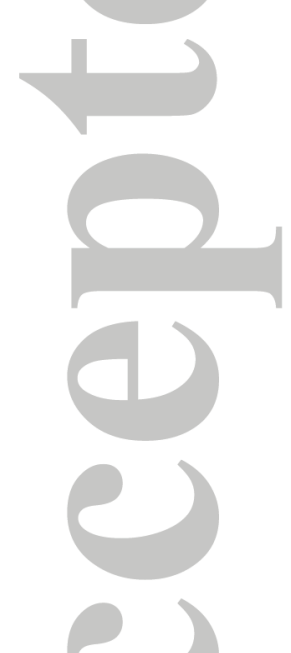


Table 4. Hazard ratios ${ }^{\mathrm{a}}$ (HRs) and $95 \%$ confidence intervals (CIs) for prostate cancer risk associated with dietary intake of fatty acids (quintiles)

\begin{tabular}{|c|c|c|c|c|c|c|c|c|c|c|c|c|}
\hline & \multicolumn{2}{|c|}{ Q1 } & \multicolumn{2}{|r|}{ Q2 } & \multicolumn{2}{|r|}{ Q3 } & \multicolumn{2}{|r|}{ Q4 } & \multicolumn{2}{|r|}{ Q5 } & \multirow{2}{*}{$\begin{array}{c}\text { Linear model }^{\mathrm{b}} \\
\text { HR }(95 \% \mathrm{CI})\end{array}$} & \multirow[t]{2}{*}{ P-trend } \\
\hline & Cases & HR & Cases & HR $(95 \%$ CI) & Cases & HR $(95 \%$ CI) & Cases & HR $(95 \%$ CI $)$ & Cases & HR $(95 \%$ CI) & & \\
\hline SFA & 91 & 1.00 & 99 & $1.02(0.72,1.45)$ & 80 & $0.81(0.57,1.17)$ & 88 & $1.04(0.72,1.51)$ & 106 & $0.98(0.68,1.41)$ & $1.00(0.89,1.12)$ & 0.96 \\
\hline Myristic acid (14:0) & 101 & 1.00 & 83 & $0.74(0.52,1.06)$ & 79 & $0.78(0.54,1.11)$ & 95 & $0.92(0.65,1.32)$ & 106 & $0.87(0.61,1.25)$ & $1.00(0.88,1.12)$ & 0.94 \\
\hline Palmitic acid (16:0) & 87 & 1.00 & 102 & $1.14(0.80,1.63)$ & 91 & $1.04(0.73,1.50)$ & 82 & $0.93(0.64,1.35)$ & 102 & $1.17(0.82,1.69)$ & $1.02(0.91,1.15)$ & 0.71 \\
\hline Stearic acid (18:0) & 90 & 1.00 & 99 & $1.21(0.85,1.71)$ & 82 & $0.88(0.61,1.27)$ & 91 & $1.04(0.72,1.49)$ & 102 & $1.02(0.71,1.46)$ & $0.99(0.88,1.11)$ & 0.82 \\
\hline MUFA & 89 & 1.00 & 105 & $1.37(0.97,1.95)$ & 102 & $1.21(0.85,1.73)$ & 92 & $1.26(0.87,1.82)$ & 76 & $1.12(0.75,1.65)$ & $1.02(0.91,1.15)$ & 0.70 \\
\hline Palmitoleic acid (16:1n-7) & 96 & 1.00 & 103 & $1.20(0.85,1.69)$ & 88 & $1.05(0.74,1.49)$ & 97 & $1.29(0.91,1.83)$ & 80 & $1.23(0.84,1.79)$ & $1.07(0.95,1.20)$ & 0.25 \\
\hline Oleic acid $(18: 1 n-9)$ & 93 & 1.00 & 100 & $1.16(0.82,1.64)$ & 95 & $1.11(0.77,1.58)$ & 99 & $1.23(0.86,1.77)$ & 77 & $1.03(0.70,1.52)$ & $1.02(0.91,1.15)$ & 0.76 \\
\hline PUFA & 77 & 1.00 & 103 & $1.50(1.04,2.16)$ & 107 & $1.57(1.09,2.25)$ & 89 & $1.26(0.86,1.84)$ & 88 & $1.19(0.81,1.75)$ & $1.01(0.90,1.13)$ & 0.86 \\
\hline n-3 PUFA & 98 & 1.00 & 107 & $1.04(0.74,1.46)$ & 109 & $1.19(0.84,1.69)$ & 63 & $0.68(0.47,1.00)$ & 87 & $1.04(0.72,1.51)$ & $0.97(0.86,1.09)$ & 0.60 \\
\hline $\operatorname{ALA}(18: 3 n-3)$ & 91 & 1.00 & 105 & $1.16(0.82,1.64)$ & 103 & $1.16(0.81,1.66)$ & 91 & $1.04(0.73,1.49)$ & 74 & $0.99(0.68,1.45)$ & $0.99(0.88,1.10)$ & 0.79 \\
\hline EPA $(20: 5 n-3)$ & 119 & 1.00 & 83 & $0.81(0.57,1.14)$ & 86 & $0.81(0.58,1.14)$ & 97 & $0.95(0.68,1.33)$ & 79 & $0.79(0.56,1.12)$ & $0.95(0.84,1.06)$ & 0.35 \\
\hline DHA (22:6n-3) & 109 & 1.00 & 89 & $0.89(0.63,1.26)$ & 94 & $0.98(0.70,1.39)$ & 89 & $0.91(0.64,1.29)$ & 83 & $0.87(0.61,1.25)$ & $0.96(0.86,1.08)$ & 0.52 \\
\hline DPA $(22: 5 n-3)$ & 100 & 1.00 & 107 & $1.25(0.89,1.77)$ & 85 & $1.06(0.74,1.51)$ & 88 & $0.97(0.68,1.38)$ & 84 & $1.00(0.70,1.42)$ & $0.97(0.86,1.08)$ & 0.55 \\
\hline n-6 PUFA & 74 & 1.00 & 104 & $1.55(1.07,2.23)$ & 108 & $1.67(1.15,2.41)$ & 89 & $1.36(0.93,2.01)$ & 89 & $1.22(0.83,1.79)$ & $1.01(0.91,1.13)$ & 0.81 \\
\hline Linoleic acid $(18: 2 n-6)$ & 74 & 1.00 & 105 & $1.58(1.10,2.28)$ & 108 & $1.70(1.18,2.46)$ & 87 & $1.36(0.92,1.99)$ & 90 & $1.24(0.85,1.82)$ & $1.01(0.91,1.13)$ & 0.81 \\
\hline DGLA (20:3n-6) & 89 & 1.00 & 106 & $1.29(0.92,1.83)$ & 90 & $1.08(0.76,1.56)$ & 76 & $0.93(0.64,1.34)$ & 103 & $1.09(0.77,1.56)$ & $0.99(0.88,1.11)$ & 0.87 \\
\hline Arachidonic acid (20:4n-6) & 88 & 1.00 & 115 & $1.38(0.98,1.94)$ & 83 & $1.13(0.78,1.64)$ & 76 & $0.93(0.64,1.35)$ & 102 & $1.25(0.87,1.79)$ & $1.03(0.92,1.15)$ & 0.64 \\
\hline n-6:n-3 PUFA & 83 & 1.00 & 85 & $1.07(0.74,1.54)$ & 79 & $1.00(0.69,1.44)$ & 109 & $1.53(1.07,2.19)$ & 108 & $1.15(0.81,1.64)$ & $1.07(0.96,1.20)$ & 0.21 \\
\hline Trans & 107 & 1.00 & 103 & $1.09(0.77,1.53)$ & 83 & $0.92(0.65,1.31)$ & 81 & $1.01(0.71,1.45)$ & 90 & $1.28(0.90,1.81)$ & $1.07(0.95,1.20)$ & 0.25 \\
\hline
\end{tabular}

Models adjusted for: country of birth, education, alcohol intake, physical activity, total energy intake from food and family history of cancer and stratified by smoking status HRs per 1 gram increment 